Accepted for publication in the Astrophysical Journal

\title{
Deposing the Cool Corona of KPD 0005+5106
}

\author{
Jeremy J. Drake ${ }^{1}$ and Klaus Werner ${ }^{2}$ \\ ${ }^{1}$ Smithsonian Astrophysical Observatory, MS-3, \\ 60 Garden Street, \\ Cambridge, MA 02138 \\ jdrake@cfa.harvard.edu \\ ${ }^{2}$ Institut für Astronomie und Astrophysik, Sand 1, 72076 Tübingen, Germany \\ werner@astro.uni-tuebingen.de
}

\begin{abstract}
The ROSAT PSPC pulse height spectrum of the peculiar He-rich hot white dwarf KPD 0005+5106 provided a great surprise when first analysed by Fleming, Werner \& Barstow (1993). It defied the best non-LTE modelling attempts in terms of photospheric emission from He-dominated atmospheres including $\mathrm{C}, \mathrm{N}$ and $\mathrm{O}$ and was instead interpreted as the first evidence for a coronal plasma around a white dwarf. We show here that a recent high resolution Chandra LETGS spectrum has more structure than expected from a thermal bremsstrahlung continuum and lacks the narrow lines of H-like and He-like $\mathrm{C}$ expected from a coronal plasma. Moreover, a coronal model requires a total luminosity more than two orders of magnitude larger than that of the star itself. Instead, the observed 20-80 $\AA$ flux is consistent with photospheric models containing trace amounts of heavier elements such as Fe. The soft X-ray flux is highly sensitive to the adopted metal abundance and provides a metal abundance diagnostic. The weak X-ray emission at $1 \mathrm{keV}$ announced by O'Dwyer et al (2003) instead cannot arise from the photosphere and requires alternative explanations. We echo earlier speculation that such emission arises in a shocked wind. Despite the presence of UV-optical O VIII lines from transitions between levels $n=7-10$, no X-ray O VIII Ly $\alpha$ flux is detected. We show that O VIII Lyman photons can be trapped by resonant scattering within the emitting plasma and destroyed by photoelectric absorption.
\end{abstract}

Subject headings: stars: activity — stars: coronae — stars: white dwarfs — stars: mass loss - X-rays: stars 


\section{Introduction}

The hot $10^{6}-10^{7} \mathrm{~K}$ coronae on the Sun and other late-type stars are believed to be sustained by mechanical energy in their outer convection zones, which is dissipated at the surface through the medium of magnetic fields generated and amplified by differential rotation and convection in the interior. This paradigm is reinforced by the decline and disappearance of $\mathrm{X}$-ray emission that occurs toward hotter stars at spectral type A, which also corresponds to the disappearance of outer convection zones. While the extensive Einstein stellar survey (Vaiana et al. 1981) detected copious X-rays from earlier O- and B-type stars in addition to later types, this has generally been attributed to emission from radiatively-driven supersonic shock-heated winds rather than coronal plasma (Cassinelli 1982), although the possible contributions from magnetically-confined plasma have recently been reconsidered based on recent Chandra and XMM-Newton high resolution spectra (e.g. Miller 2002).

In the above context, the low resolution ROSAT Position-Sensitive Proportional Counter (PSPC) pulse height spectrum of the unusual hot He-rich white dwarf KPD 0005+5106 (Downes et al. 1985, WD 0005+511) presented a major surprise. Fleming et al. (1993) found that they could not match this spectrum using the best available spectral models computed using non-LTE model atmospheres containing $\mathrm{He}, \mathrm{C}, \mathrm{N}$ and O. Instead, they found that a satisfactory fit to the data could be obtained from thermal bremsstrahlung and optically-thin plasma models with temperatures of $2-3 \times 10^{5} \mathrm{~K}$. The apparent impropriety of photospheric spectral models and success of thermal plasma models provided evidence that the X-rays originated not from deeper atmospheric layers but from a coronal plasma encircling the star. KPD $0005+5106$ then became the first white dwarf thought to have a corona, albeit a cool one compared to the $10^{6}-10^{7} \mathrm{~K}$ coronae of late-type stars. As Fleming et al. (1993) noted, this result was quite novel and surprising, since all previously observed X-ray emission from white dwarfs had been photospheric in nature. Moreover, while DA and DB stars can possess significant convection zones corresponding to $\mathrm{H}$ and He ionization layers and might conceivably sustain magnetic dynamo activity, this is not the case for the much hotter $1.2 \times 10^{5} \mathrm{~K}$ effective temperature of KPD $0005+5106$ (Werner et al. 1994) where He is completely ionized.

A corona about KPD $0005+5106$ is perhaps a less surprising proposition when viewed in the light of other peculiarities of its spectrum. Emission lines of $\mathrm{C} \mathrm{V}, \mathrm{N}$ V and O VIII have been detected at UV and optical wavelengths, prompting the suggestion of ongoing, and possibly shock-heated, mass loss (Werner et al. 1994; Sion \& Downes 1992; Sion et al. 1997). More recently, O'Dwyer et al. (2003) and (see also Chu et al. 2004) have reported a detection of weak X-ray emission at $1 \mathrm{keV}$ based on a re-analysis of deeper ROSAT PSPC observations of KPD 0005+5106, while Otte et al. (2004) have presented the discovery of an 
O VI-emitting nebula associated with the object.

In this paper, however, we show that the Chandra Low Energy Transmission Grating Spectrograph and High Resolution Camera Spectroscopic detector (LETG+HRC-S) spectrum of KPD 0005+5106 can be qualitatively modelled as photospheric emission, and does not require a coronal explanation. The excess flux found by Fleming et al. (1993) in the 0.2$0.3 \mathrm{keV}$ range arises from deeper atmospheric layers revealed by a lower continuous opacity in this region. Difficulties with earlier photospheric modelling attempts are shown to have arisen because of the neglect of trace heavier elements such as Fe. The $1 \mathrm{keV}$ emission found by O'Dwyer et al. (2003) lies below our detection threshold, but cannot be explained by photospheric models.

\section{Observations and Analysis}

KPD 0005+5106 was observed by Chandra using the LETG+HRC-S in its standard configuration on 2000 October 25 between UT 05:16 and 10:58 for a total of 19114s, after correction for instrument deadtime and bad event filtering.

Initial reduction of satellite telemetry was performed by the Chandra X-ray Center Standard Data Processing software, but was completely reprocessed by us using CIAO 3.1. The analysis described here is based on the Level 2 products of this reprocessing. The scientific analysis was undertaken using the PINTofALE ${ }^{1}$ IDL $^{2}$ software suite (Kashyap \& Drake 2000). The spectrum of KPD $0005+5106$ was extracted by summing the events within a window $3.6^{\prime \prime}$ in width in the cross-dispersion direction, and background was estimated in two strips of width $18^{\prime \prime}$ each located on either side of the spectral trace. The final extracted spectrum, binned at $5 \AA$ intervals, is illustrated in Figure 1. The distribution of the dispersed photon events is consistent with a continuum source: no narrow emission line features were discernible.

Our analysis involved comparison of the observed LETGS spectrum of KPD 0005+5106 with the emergent spectra computed for a range of line blanketed non-LTE model atmospheres and for optically-thin collision-dominated coronal models. Coronal radiative loss models were computed using PINTofALE, employing line and continuum emissivities computed using atomic data from the CHIANTI database version 4.02 (Young et al. 2003) and the ionization equilibrium of Mazzotta et al. (1998).

\footnotetext{
${ }^{1}$ Freely available from http://hea-www.harvard.edu/PINTofALE/

${ }^{2}$ Interactive Data Language, Research Systems Inc.
} 
Model atmosphere computations were performed using the PRO2 code (Werner et al. 2003) assuming hydrostatic and radiative equilibrium. Model atoms and atomic data used for detailed non-LTE calculations have been described recently by Werner et al. (2004). Constraints for the input model parameters are provided by earlier analyses of UV spectra obtained by the HST Faint Object Spectrograph (FOS) and Goddard High Resolution Spectrograph (GHRS), and optical spectra obtained at the $3.5 \mathrm{~m}$ telescope at Calar Alto (Werner et al. 1994, 1996). Additionally, we have examined more recent Far Ultraviolet Spectroscopic Explorer (FUSE) spectra of KPD 0005+5106 to provide further constraints on the abundances of the elements $\mathrm{O}$ and Fe that exhibit lines in the FUSE bandpass. From the lack of Fe lines an upper abundance limit was determined by Miksa et al. (2002). From O VI lines we have determined the $\mathrm{O}$ abundance with the models computed for the present paper. Our adopted nominal parameters for KPD 0005+5106 are listed in Table 1. For a single test calculation including sulfur we assume a solar S abundance (by mass fraction). The absolute flux calibration for our models is provided by HST UV observations to high precision, and for comparisons with Chandra spectra we normalise the models to the observed flux density of $4.0 \times 10^{-12} \mathrm{erg} \mathrm{cm}^{-2} \mathrm{~s}^{-1} \AA^{-1}$ at $1200 \AA$.

The reasoning of Fleming et al. (1993) leading to the conclusion that KPD 0005+5106 has a corona was based on the comparison of low resolution X-ray pulse height spectra with model predictions for atmospheres containing $\mathrm{C}, \mathrm{N}$ and $\mathrm{O}$. We argue in this paper that difficulties in matching the X-ray data with photospheric models was primarily due to the neglect of heavier elements that can contaminate the photosphere. While settling under the strong gravity of the white dwarf tends to empty the atmosphere of heavier elements, the atmospheres of hotter stars can be substantially enriched by radiative levitation (e.g. Chayer et al. 1995; Schuh et al. 2002).

Model fluxes for atmospheres with an effective temperature $T_{\text {eff }}=120000 \mathrm{~K}$, with and without Fe, are compared in Figure 2. Models include full non-LTE treatment of $\mathrm{C}, \mathrm{O}, \mathrm{Ne}, \mathrm{S}$ and Fe, whose abundances were adopted from Table 1. Also illustrated is the blackbody flux density distribution for the same temperature. It is interesting to note that the blackbody has much less short wavelength flux than do the more realistic models: the short wavelength extension of the spectra of the latter arises because of changes in the opacity with wavelength that expose the deeper, hotter atmospheric layers at these wavelengths. Addition of Fe with an abundance equal to the best current upper limit (Table 1) however, dramatically reduces the soft X-ray flux: clearly any photospheric soft X-ray emission from KPD 0005+5106 and similar stars is critically dependent on the photospheric abundances of heavier elements, as was also demonstrated earlier in the case of EUV fluxes at longer wavelengths for slightly cooler stars (e.g. Barstow et al. 1995). 
Coronal and photospheric model spectra are compared to the observations in Figure 3. Coronal models computed for the He-rich composition listed in Table 1 are dominated by the He bound-free and free-free continua, and by the lines of He-like C near $40 \AA$. While it is tempting to interpret the peak in the observed flux between 40 and $45 \AA$ as being due to these $\mathrm{C}$ V lines, there are no such lines visible in the spectrum where the observed counts are more smoothly distributed. The absence of $\mathrm{C} \mathrm{V}$ lines does not necessarily argue against the coronal interpretation of the spectrum, however, since one might expect heavier elements to gravitationally settle out of such a plasma. We therefore also computed models in which the metal abundances were reduced by a factor of 100 .

We confirm the result of Fleming et al. (1993) that the best-fit coronal spectrum has a temperature in the range $2-3 \times 10^{5} \mathrm{~K}$; our best-fit model corresponds to $T=2 \times 10^{5} \mathrm{~K}$, with an interstellar absorption component represented by a column of neutral hydrogen of $3.5 \times 10^{20} \mathrm{~cm}^{-2}$. Qualitative comparison of the best-fit coronal model and data suggest, however, that the latter have a more complex shape than can be achieved by the former.

Photospheric models both with and without Fe were computed, as was a test model including S; a small subsection of the models investigated are illustrated in the lower panel of Figure 3. It is clear that models with only $\mathrm{C}, \mathrm{O}$ and Ne greatly overpredict the observed X-ray flux by factors of 10 or more. Based on Figure 2, we see that the emergent soft Xray flux can be reduced by addition of $\mathrm{Fe}$ and the opacity this element provides at these wavelengths. This is borne out in practice, and the photospheric spectrum that was found to best match the observations corresponds roughly to the parameters $T_{\text {eff }}=120000 \mathrm{~K}$ and $\mathrm{Fe} / \mathrm{He}=-5.5$, with a neutral hydrogen column density of $3 \times 10^{20} \mathrm{~cm}^{-2}$. It is clear from Figure 3 that there is no obvious reason to prefer coronal models over photospheric ones.

While we have not succeeded in producing a photospheric model that perfectly matches the observations, we have achieved a good qualitative match by adding only plausible amounts of $\mathrm{Fe}$ to the $\mathrm{C}, \mathrm{O}$ and $\mathrm{Ne}$ abundance mixture. Trace amounts of other metals such as Na, Mg, S, Ar, Ca and Ni could also affect significantly the soft X-ray spectrum, and we could doubtless obtain a better match through their addition and subsequent optimisation of other parameters such as effective temperature, surface gravity and ISM absorption. The test model including S, for example, introduced an absorption edge near $43 \AA$ with little effect on the emergent flux at longer wavelengths; careful adjustment of the $\mathrm{S}$ abundance could aid in a better prediction of the observed drop in flux between 40 and $45 \AA$. Further progress in this direction would demand some additional constraints on the abundances of these other metals in order to limit the available parameter space to tractible proportions. 


\section{Discussion and Conclusions}

\subsection{Photospheric vs Coronal Emission}

We have shown that the Chandra LETG+HRC-S spectrum of KPD 0005+5106 in the 25-90 A range can be qualitatively modelled by photospheric emission from an atmosphere containing $\mathrm{He}, \mathrm{C}, \mathrm{O}$ and $\mathrm{Ne}$ in expected amounts, with the addition of $\mathrm{Fe}$ at a level consistent with the current observational upper limit. With these quite plausible models, there is no requirement in the data to resort to more exotic solutions such as a coronal model. The quality of the current short LETGS exposure does not allow us to rule out a significant amount of coronal emission with a high degree of confidence, though the best fit model based on purely coronal emission is statistically unacceptable with a reduced $\chi^{2}=2.1$.

We can, however, completely discount a coronal interpretation of the ROSAT and Chan$d r a$ spectra on energetic grounds. A coronal plasma model at a temperature of $2-3 \times 10^{5} \mathrm{~K}$ absorbed by an ISM column of $N_{H} \sim 10^{20} \mathrm{~cm}^{-2}$ and normalised to the observed ROSAT spectrum has a total luminosity 2-3 orders of magnitude greater than than the star itself. This is qualitatively illustrated in Figure 2, where the coronal model is shown normalised relative to the photospheric models to the approximate relative level required to explain the observed 20-80 A X-ray flux.

\subsection{Remaining Puzzles}

Despite the ability of photospheric models to explain the Chandra LETGS spectrum of KPD $0005+5106$ and the energetic impossibility of a coronal explanation, there are two additional puzzles regarding the spectrum of this object at both shorter and longer wavelengths that cannot be explained by our photospheric models. These are the detection of flux at $\sim 1 \mathrm{keV}(12.4 \AA$ ) by O'Dwyer et al. (2003), and the observation of $\Delta n=1$ emission lines of O VIII between levels $n=7-10$ at UV and optical wavelengths (Werner et al. 1994; Sion \& Downes 1992; Sion et al. 1997). It seems likely that the two are related.

We have verified that there is no trace of any flux near $12 \AA$ in the LETGS spectrum, although the flux density reported by O'Dwyer et al. (2003) based on ROSAT observations (5 count $\mathrm{s}^{-1}$ in the Boron filter) lies below our LETGS detection limit in this short observation: only $\sim 18$ LETG+HRC-S counts are expected, spread over a fairly large detector area over which the background is an order of magnitude larger.

More puzzling is the lack of O VIII lines in the Chandra data: the excited higher $n$ states

will decay through cascades to lower $n$, and we would expect to see some fraction of this 
decay channel in the Lyman series resonance lines. Based on the Goddard High Resolution Spectrograph UV spectrum of KPD 0005+5106 obtained on 1994 June 1 and analysed by Werner et al. (1996), the O VIII $2976.57 \AA n=8-7$ transition flux is $0.07 \mathrm{ph} \mathrm{cm}^{-2} \mathrm{~s}^{-1}$. The upper limit to the flux of the O VIII $18.98 \AA n=2-1$ doublet in the Chandra spectrum is more than three orders of magnitude less than this.

There are two possible explanations for the absence of the O VIII Ly $\alpha$ lines: (1) the source is variable and during the Chandra observation the O VIII lines were much weaker; (2) the X-ray lines are suppressed by some mechanism. While the lack of any simultaneous observations accompanying the Chandra pointing prevents us drawing definitive conclusions regarding (1), we note that optical spectra of KPD $0005+5106$ similar to those described by Werner et al. (1994) and obtained at the Calar Alto 3.5m telescope in 1991 July, 1992 September and 1994 May, exhibit identical O VIII $n=10-9$ and 9-8 lines and conclude that large amplitude variability in O VIII is unlikely. Instead, the most likely explanation for the lack of prominent X-ray O VIII lines is that they are suppressed.

We speculate that the O VIII Ly $\alpha$ lines are formed in a low density medium or wind surrounding the white dwarf, as has been suggested by earlier workers (Sion \& Downes 1992; Sion et al. 1997; Werner et al. 1994). In this case, the low $n$ resonance lines might be sufficiently optically thick to resonance scattering that line photons are destroyed by photoelectric absorption as they undergo multiple scattering events within the emitting region. The line centre optical depth, $\tau$, in a He-dominated low-density medium can be written (e.g., Acton 1978; Mariska 1992):

$$
\tau=1.16 \cdot 10^{-14} \cdot \frac{n_{\mathrm{i}}}{n_{\mathrm{el}}} A_{\mathrm{Z}} \frac{n_{\mathrm{H}}}{n_{\mathrm{e}}} \lambda f \sqrt{\frac{M}{T}} n_{\mathrm{e}} \ell
$$

for ion fraction $\left(n_{\mathrm{i}} / n_{\mathrm{el}}\right.$, element abundance $A_{\mathrm{Z}}$, oscillator strength $f$, temperature $T$, electron density $n_{\mathrm{e}}$, atomic weight $M$, and where $n_{\mathrm{He}} / n_{\mathrm{e}} \sim 0.5$, and $\ell$ is the total path length along the line of sight through the emitting plasma.

Taking O VIII $18.97 \AA 2 p \rightarrow 1 s$ as an example, we have estimated the optical depth for two limiting cases of the morphology of the emitting plasma: (a) a "corona" in hydrostatic equilibrium, for which the scale height, or typical pathlength, $\ell$, is much smaller than the stellar radius, $\ell<<R_{\star}$; (b) an extended wind for which $\ell>>R_{\star}$. We have used PINTofALE (Kashyap \& Drake 2000) to estimate the total volume emission measure, $n_{e}^{2} V$, of the O VIII emitting plasma based on the total of 25 ROSAT (Boron filter) counts observed near $1 \mathrm{keV}$ in $5 \mathrm{ks}$ by O'Dwyer et al. (2003). We assumed that the plasma is isothermal with $T=2 \times 10^{6} \mathrm{~K}$ (corresponding to the peak in the $\mathrm{O}^{7+}$ ion fraction, such that $n_{\mathrm{i}} / n_{\mathrm{el}} \sim 1$ ), is collisiondominated, has a He-dominated composition as listed in Table 1 and is absorbed by an ISM column density represented by a neutral $\mathrm{H}$ column of $N_{H}=5 \times 10^{20} \mathrm{~cm}^{-2}$. For the 
approximate distance of 270 pc estimated by Werner et al. (2004), the observed ROSAT counts imply a volume emission measure $n_{e}^{2} V \sim 10^{54} \mathrm{~cm}^{-3}$.

Adopting the stellar parameters of KPD 0005+5106 from Werner et al. (1994), the pressure scale height for a He plasma in hydrostatic equilibrium, $k T / m_{H e} g_{\star}=6.2 \times 10^{6} \mathrm{~cm}$, or $0.2 \%$ of the stellar radius and the plasma density is $\sim 10^{14} \mathrm{~cm}^{-3}$. Based on Equation 1, the optical depth in O VIII Ly $\alpha$ is $\tau=17$-ie extremely large. For a much more extended plasma, the line centre optical depth is $\tau \sim 200$ for $\ell \sim 10 R_{\star}$ and remains very large $(\tau>10)$ for emitting size scales up to $10^{4} R_{\star}$.

The large optical depths in the O VIII Ly $\alpha$ resonance line imply that these line photons are trapped within the plasma and must undergo many scattering events before escaping. If the plasma has a temperature of $\sim 2 \times 10^{6} \mathrm{~K}$, it will contain a significant fraction of H-like and He-like ions among the light elements $\mathrm{C}, \mathrm{N}$ and $\mathrm{O}$, together with ions with $n=3$ ground states of elements such as Fe if these are present. The total photoelectric absorption cross-section for our postulated plasma, computed using the Hartree-Dirac-Slater photoionisation cross-sections of Verner et al. (1993) and Verner \& Yakovlev (1995), together with ion fractions from Mazzotta et al. (1998), amounts to $\sigma \sim 10^{-23} \mathrm{~cm}^{2}$. For the plasma radial extents considered above, for which O VIII Ly $\alpha$ is optically thick, the typical column density of the plasma in terms of He nuclei ranges from several $10^{20}$ to $10^{22} \mathrm{~cm}^{-2}$. Absorption for weaker, optically thin lines and continuum is therefore negligible. However, the trapped O VIII line photons must undergo such a large number of scattering events that they are destroyed by photoelectric absorption before they can escape. Similar arguments apply to higher Lyman series lines; while their f-values are smaller, they are also effectively quenched through decays to levels $n>1$ and then eventually through Ly $\alpha$ as soon as scattering depths become significant. O VIII $16.01 \AA \mathrm{Ly} \beta$, for example, is lost to resonant scattering in decays through the $n=3-2$ Balmer line at $102.43 \AA$, and then through Ly $\alpha$. Unfortunately, the ISM absorbing column to KPD $0005+5106$ is too large for the Balmer lines to be visible, though a future detection of O VIII Ba $\alpha$ in other similar objects to KPD 0005+5106 would provide a useful test of this model.

If the $\sim 1 \mathrm{keV}$ emission found by O'Dwyer et al. (2003) is indeed due to a wind or outflow, then an optically-thin plasma model with a temperature of $2 \times 10^{6} \mathrm{~K}$ accounting for the observed ROSAT counts at this energy has a total luminosity of $\sim 5 \times 10^{31} \mathrm{erg} \mathrm{s}^{-1}$, or $10^{-4}$ times the stellar bolometric luminosity. The 0.1-2.5 keV X-ray to bolometric luminosity ratio is $\sim 2 \times 10^{-5}$-a somewhat higher value than observed for OB stars, whose X-rays are believed to originate in shocked winds and for which the X-ray to bolometric luminosity ratio $L_{X} / L_{b o l} \sim 10^{-6}-10^{-8}$ (e.g. Berghoefer et al. 1997). 


\section{Conclusions}

We have shown that a coronal plasma is unable to match the Chandra LETG+HRC-S 20-80 A spectrum of KPD 0005+5106 and that coronal models are energetically implausible as an origin for this observed soft X-ray flux. This part of the soft X-ray spectrum of KPD $0005+5106$ can instead be explained by photospheric models containing trace amounts of heavier elements. Photospheric models do not, however, explain the soft X-ray emission at shorter wavelengths ( $\sim 12 \AA ; 1 \mathrm{keV})$ revealed recently by O'Dwyer et al. (2003) and discussed in more detail by $\mathrm{Chu}$ et al. (2004). The origin of this emission remains mysterious, though an outflow or wind with a temperature of $\sim 2 \times 10^{6} \mathrm{~K}$ is able to explain both the X-rays and the presence of high $n$ O VIII UV-optical emission lines but an absence of significant O VIII Ly $\alpha$ flux in the Chandra spectrum. In this scenario, the Ly $\alpha$ photons are trapped by resonance scattering and destroyed by photoelectric absorption. If an outflow is responsible for the $1 \mathrm{keV} \mathrm{X}$-rays, the total luminosity of this plasma amounts to $\sim 10^{-4} L_{b o l}$, which is a factor of 100 or so larger than for winds of OB stars.

We thank the NASA AISRP for providing financial assistance for the development of

the PINTofALE package, and the CHIANTI project for making publicly available the results of their substantial effort in assembling atomic data useful for coronal plasma analysis. Brad Wargelin is thanked for useful comments. JJD was supported by NASA contract NAS839073 to the Chandra X-ray Center during the course of this research. X-ray data analysis in Tübingen is supported by the DLR under grant 50 OR 0201.

\section{REFERENCES}

Acton, L. W. 1978, ApJ, 225, 1069

Barstow, M. A., Holberg, J. B., Werner, K., \& Nousek, J. A. 1995, Advances in Space Research, 16, 73

Berghoefer, T. W., Schmitt, J. H. M. M., Danner, R., \& Cassinelli, J. P. 1997, A\&A, 322, 167

Cassinelli, J. P. 1982, Advances in Space Research, 2, 67

Chayer, P., Fontaine, G., \& Wesemael, F. 1995, ApJS, 99, 189

Chu, Y., Gruendl, R. A., Williams, R. M., Gull, T. R., \& Werner, K. 2004, AJ, 128, 2357

Downes, R. A., Liebert, J., \& Margon, B. 1985, ApJ, 290, 321 
Fleming, T. A., Werner, K., \& Barstow, M. A. 1993, ApJ, 416, L79+

Kashyap, V. L. \& Drake, J. J. 2000, Bulletin of the American Astronomical Society, 32, 1227

Mariska, J. T. 1992, The solar transition region (Cambridge Astrophysics Series, New York: Cambridge University Press, —c1992)

Mazzotta, P., Mazzitelli, G., Colafrancesco, S., \& Vittorio, N. 1998, A\&AS, 133, 403

Miksa, S., Deetjen, J. L., Dreizler, S., Kruk, J. W., Rauch, T., \& Werner, K. 2002, A\&A, 389,953

Miller, N. A. 2002, in ASP Conf. Ser. 277: Stellar Coronae in the Chandra and XMMNEWTON Era, ed. F. Favata \& J. J. Drake, San Francisco, 379

O'Dwyer, I. J., Chu, Y., Gruendl, R. A., Guerrero, M. A., \& Webbink, R. F. 2003, AJ, 125, 2239

Otte, B., Dixon, W. V. D., \& Sankrit, R. 2004, ApJ, 606, L143

Schuh, S. L., Dreizler, S., \& Wolff, B. 2002, A\&A, 382, 164

Sion, E. M. \& Downes, R. A. 1992, ApJ, 396, L79

Sion, E. M., Holberg, J. B., Barstow, M. A., \& Scheible, M. P. 1997, AJ, 113, 364

Vaiana, G. S., Cassinelli, J. P., Fabbiano, G., Giacconi, R., Golub, L., Gorenstein, P., Haisch, B. M., Harnden, F. R., Johnson, H. M., Linsky, J. L., Maxson, C. W., Mewe, R., Rosner, R., Seward, F., Topka, K., \& Zwaan, C. 1981, ApJ, 245, 163

Verner, D. A. \& Yakovlev, D. G. 1995, A\&AS, 109, 125

Verner, D. A., Yakovlev, D. G., Band, I. M., \& Trzhaskovskaya, M. B. 1993, Atomic Data and Nuclear Data Tables, 55, 233

Werner, K., Deetjen, J. L., Dreizler, S., Nagel, T., Rauch, T., \& Schuh, S. L. 2003, in ASP Conf. Ser. 288: Stellar Atmosphere Modeling, 31-1

Werner, K., Dreizler, S., Heber, U., Rauch, T., Fleming, T. A., Sion, E. M., \& Vauclair, G. 1996, A\&A, 307, 860

Werner, K., Heber, U., \& Fleming, T. 1994, A\&A, 284, 907

Werner, K., Rauch, T., Barstow, M. A., \& Kruk, J. W. 2004, A\&A, 421, 1169 
Young, P. R., Del Zanna, G., Landi, E., Dere, K. P., Mason, H. E., \& Landini, M. 2003, ApJS, 144, 135 


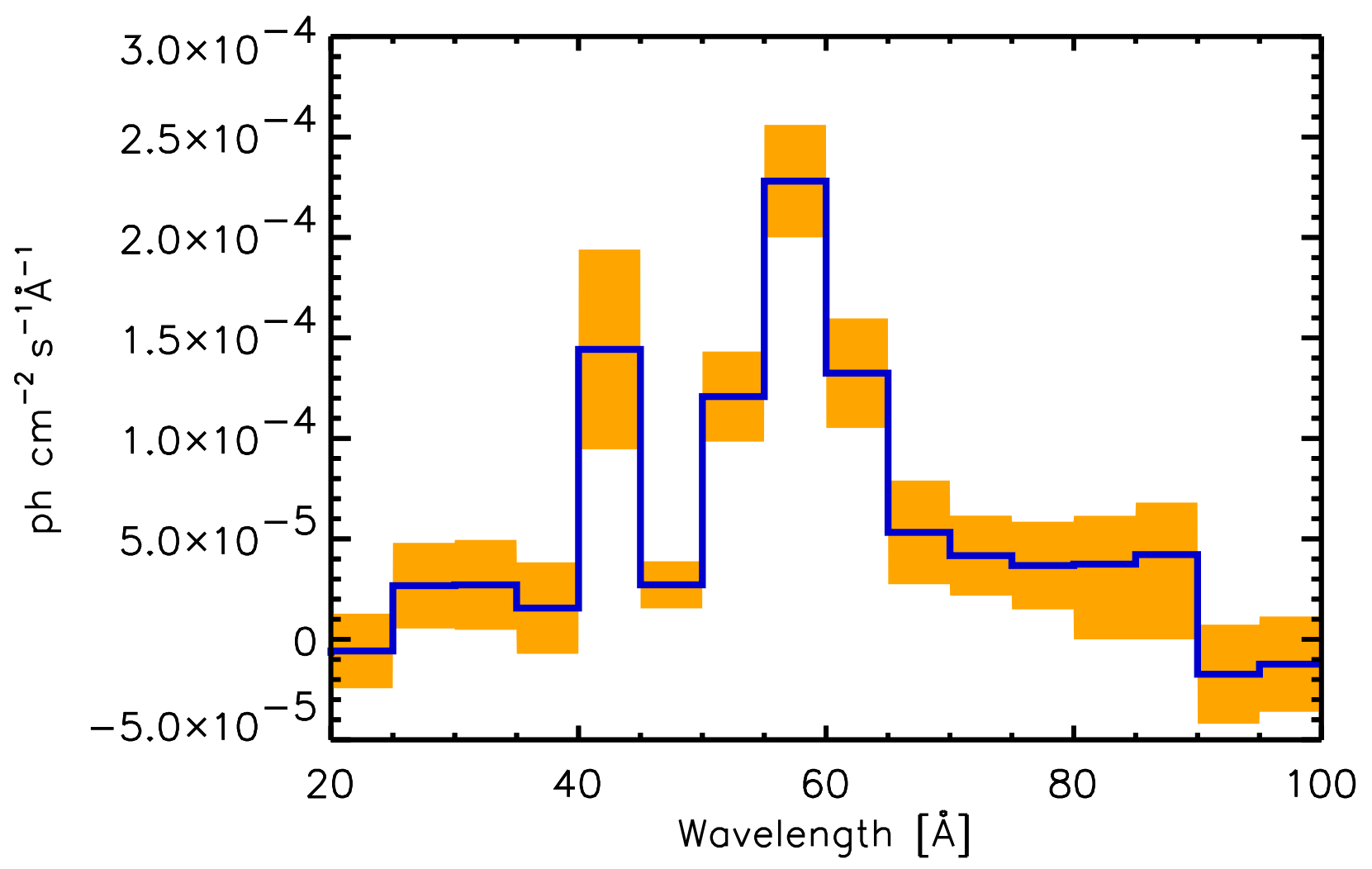

Fig. 1. - The Chandra LETG+HRC-S spectrum of KPD 0005+5106; shaded regions represent $1 \sigma$ uncertainties. No evidence for narrow lines is present in the data. We therefore show here the spectrum binned at $5 \AA$ intervals in order to illustrate the observed continuum structure. 


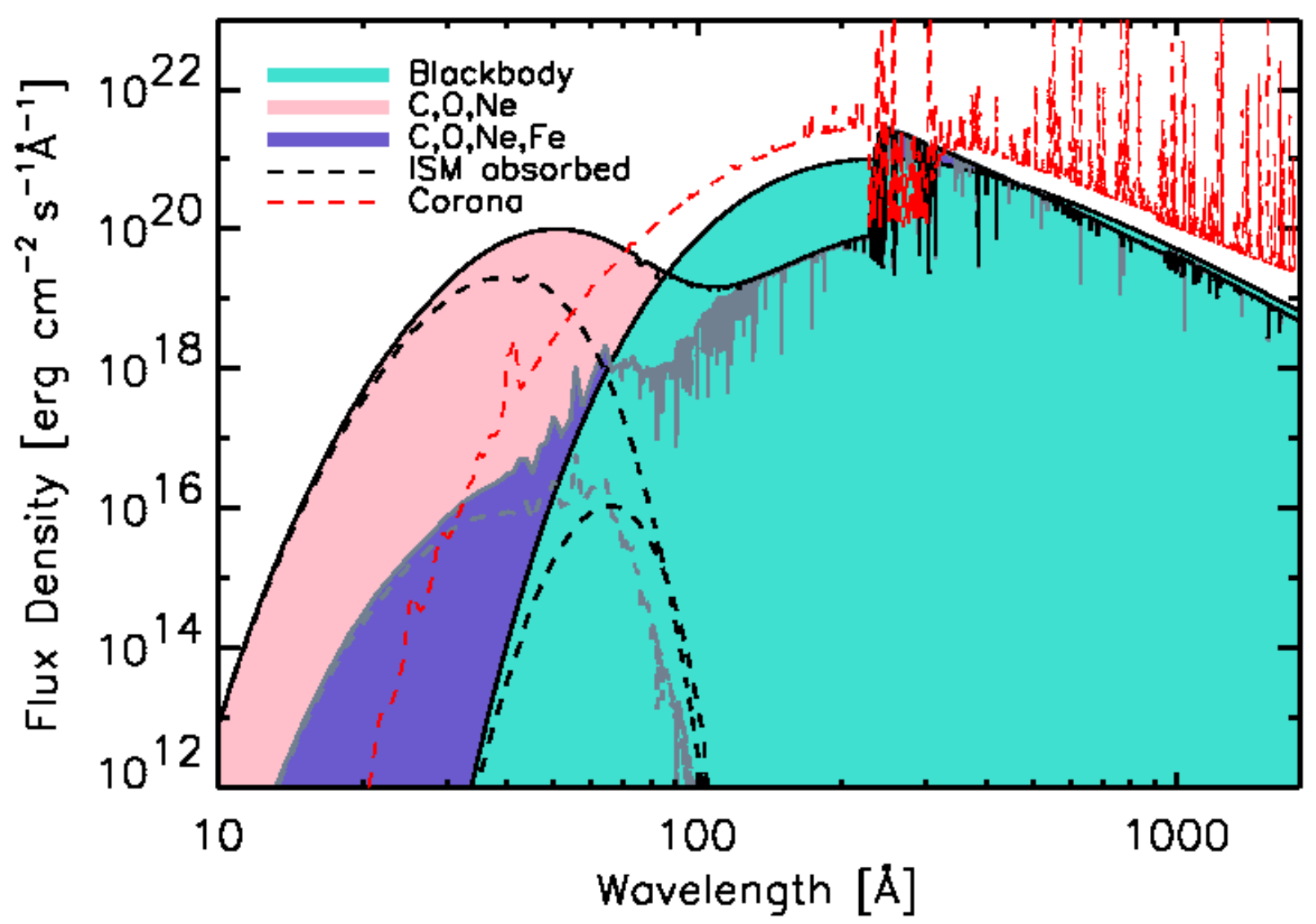

Fig. 2.- Emergent spectra in the UV-X-ray range for non-LTE model atmospheres computing using the PRO2 code (Werner et al. 2003) for the parameters and abundances listed in Table 1. Dashed curves illustrate the spectra absorbed by an intervening ISM column of $4 \times 10^{20} \mathrm{~cm}^{-2}$. The model including $\mathrm{Fe}$ assumed $\mathrm{Fe} / \mathrm{He}=-5$, consistent with the current upper limit based on UV and optical spectra. Also shown is a coronal model computed for a temperature $T=2 \times 10^{5} \mathrm{~K}$, normalised to the relative level required to explain the ROSAT observations presented by Fleming et al. (1993). 

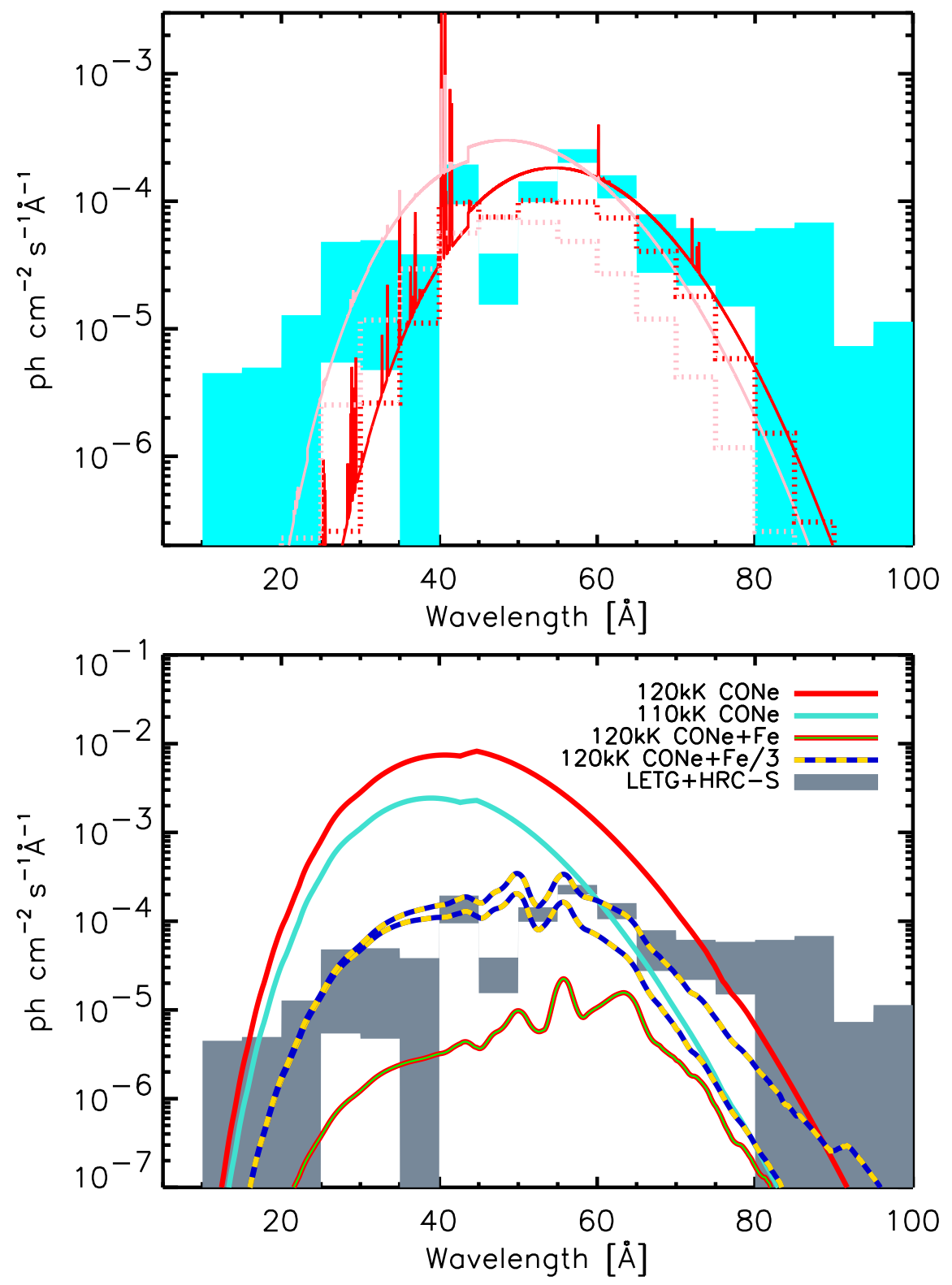

Fig. 3.- Comparison of the observed LETGS spectra (shaded region) with different coronal (upper) and non-LTE photospheric model (lower) predictions. Coronal models are shown both at the theoretical resolution of the LETGS, and binned at the same $5 \AA$ intervals as the observations. Models are attenuated by an ISM absorbing column of $N_{H}=4 \times 10^{20}$. Also shown in the lower panel is the "best fit" model (" $120 \mathrm{kK} \mathrm{CONe}+\mathrm{Fe} / 3$ ") for which $N_{H}=3 \times 10^{20}$. 
Table 1: Fundamental Parameters and Abundances for KPD 0005+5106

\begin{tabular}{lc}
\hline$T_{\text {eff }}$ & $120000 \pm 10000 \mathrm{~K}$ \\
$\log g$ & $7.0 \pm 0.5$ \\
$\mathrm{H} / \mathrm{He}$ & $\leq-0.7$ \\
$\mathrm{C} / \mathrm{He}^{\mathrm{a}}$ & $-3 \pm 0.5$ \\
$\mathrm{O} / \mathrm{He}$ & $-3.8 \pm 0.5$ \\
$\mathrm{Ne} / \mathrm{He}$ & $\leq-8$ \\
$\mathrm{~S} / \mathrm{He}$ & -4.4 \\
$\mathrm{Fe} / \mathrm{He}$ & $\leq-5$ \\
$N_{H}$ & $(5 \pm 1.1) \times 10^{20} \mathrm{~cm}^{-2}$ \\
$F_{1200}{ }^{\mathrm{b}}$ & $4.0 \times 10^{-12}$ \\
\hline
\end{tabular}

${ }^{a} \mathrm{X} / \mathrm{He}=\log _{10} n(X) / n(\mathrm{He})$

${ }^{b}$ Flux density at $1200 \AA$ in units of $\operatorname{erg~cm}^{-2} \mathrm{~s}^{-1} \AA^{-1}$ 